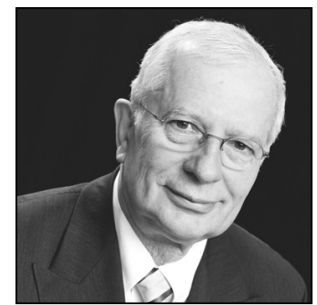

Christian Tomuschat

Professor emeritus

Humboldt University

\title{
Die Zukunft des Völkerrechts
}

\section{Vorbemerkung}

Das Völkerrecht unserer Tage hat einen hohen Entwicklungsstand erreicht. Als Netz normativer Leitaussagen verbindet es nicht nur die Staaten des Erdballs miteinander, sondern hat seinen Geltungsanspruch gleichzeitig stark über den ursprünglichen Adressatenkreis hinaus ausgeweitet. Völkerrechtliche Regeln richten sich auch an internationale Organisationen, statten Einzelpersonen mit Rechten aus oder erlegen ihnen Pflichten auf und dringen teilweise auch in die Privatrechtsordnung ein. Auch inhaltlich hat das Völkerrecht seit der Gründung der Vereinten Nationen eine neue Qualität gewonnen. War es zunächst den historischen Zufälligkeiten folgend ohne systematischen Bauplan über Jahrhunderte hinweg bruchstückhaft gewachsen, hat es seit dem Jahre 1945 Leitprinzipien gewonnen, die fundamentalen menschlichen Bedürfnissen Rechnung tragen. Frieden und Menschenrechte sind mit der UN-Charta (im Folgenden: Charta) ins Zentrum der völkerrechtlichen Ordnung gerückt.

Viele der rechtlichen Konzepte und Institutionen der Gegenwart lassen sich als unmittelbare Ableitungen aus diesen Grunddaten des internationalen Systems ableiten. Kriegs- und Interventionsverbot dienen unmittelbar der Sicherung des Friedens, das Selbstbestimmungsrecht erlaubt es jedem Volke, seine eigenen Zielvorstellungen in friedlicher Weise zu verwirklichen, und die Menschenrechte sollen nicht nur jeder menschlichen Person ein Leben in Würde und Sicherheit gewährleisten, sondern gleichzeitig auch sicherstellen, dass nicht innerstaatliche Auseinandersetzungen gewaltsam auf die internationale Ebene durchschlagen. Insgesamt hat das Völkerrecht in hohem Maße Wertvorstellungen aufgenommen, die von den Völkern der Welt weithin geteilt werden. Eine Grundsatzkritik an der bestehenden völkerrechtlichen Ordnung wird nur noch von vereinzelten Kreisen geäußert. Vor allem ein der Geschichte geschuldeter struktureller Mangel ist mittlerweile behoben worden. ${ }^{*}$ Nachdem der Prozess der Dekolonisierung seinen Abschluss gefunden hat, haben die früheren Kolonialvölker sich mit großer Hingabe an dem Prozess der Sichtung und Überprüfung des Völkerrechts beteiligt und auf diese Weise seine früheren Einseitigkeiten beseitigt. ${ }^{*}$

So ist mittlerweile ein Normengerüst entstanden, das trotz seiner Auffächerung in viele Spezialdisziplinen als eine geschlossene systematische Einheit betrachtet werden darf. Über viele Jahre hinweg hat das Gerede über die Fragmentierung des Völkerrechts die literarischen Debatten beherrscht ${ }^{*} 3$ - doch prinzipiell

1 Die von den Anhängern der Third World Approaches to International Law (TWAIL) geäußerte Kritik, vgl. etwa B.S. Chimni, 'Critical Theory of Economic Law: a Third World Approach to International Law (TWAIL) Perspective'; in: John Linarelli (ed.), Research Handbook on Global Justice and International Economic Law (Cheltenham, UK: Edward Elgar, 2013) 251273), hat sich mittlerweile überlebt und wird nur noch ideologisch gestützt.

2 Eines der Vehikel dieses tiefgreifenden Wandels ist bis zum heutigen Tage die UN-Völkerrechtskommission (International Law Commission, ILC), ein Unterorgan der UN-Generalversammlung (GV).

3 S. dazu den Bericht der Studiengruppe der Völkerrechtskommission, Yearbook of the International Law Commission 2006, Vol. II Part Two, UN-Dok. A/CN.4/SER.A/2006/Add.1 (Part 2), 176. 
zu Unrecht. ${ }^{*}$ Es ist selbstverständlich, dass sich zu eigenständigen Themenkomplexen besondere Regeln ausbilden müssen. Sowohl das Seerecht wie das Umweltrecht oder auch das Investitionsschutzrecht brauchen jeweils sachangepasste Rechtsregime, die jeweils aus den spezifischen Eigenheiten der Materie entwickelt werden müssen. Zusammengehalten werden aber diese unterschiedlichen Rechtsregime durch die allgemeinen Rechtsfiguren des Völkerrechts, vor allem durch die genannten Leitprinzipien, die der internationalen Ordnung ein festes Rahmenwerk verleihen. Was das materielle Recht angeht, lassen sich kaum noch irgendwelche Lücken grundlegender Art feststellen, auch wenn das Rechtsgebäude nach Maßgabe der tatsächlichen Entwicklungen immer wieder überprüft und nachgebessert werden muss.

Ergänzend sei sogleich festgestellt, dass das klassische zwischenstaatliche Recht nicht das Ganze der grenzüberschreitenden Regelungen ausmacht. Nichtstaatliche Akteure haben vielfach Machtpositionen errungen, die faktisch denen kleinerer Länder kaum nachstehen. Zunehmend muss sich vor allem das humanitäre Recht mit den Gewalttaten von ideologiegeprägten Extremistengruppen im innerstaatlichen Raum auseinandersetzen. Auch das Privatrecht hat sich über die Jahrzehnte hinweg vor allem seit dem Ende des Zweiten Weltkrieges weitgehend internationalisiert. In Ausübung der allgemeinen Freiheitsrechte haben Einzelmenschen und Unternehmen ein Netz von transnationalen Beziehungen gespannt, das sich neben dem zwischenstaatlichen Völkerrecht mächtig entfaltet. Die Staaten sind demgemäß in den internationalen Beziehungen nicht mehr die einzigen bedeutsamen Akteure. Ihre Monopolstellung, wenn es sie jemals gegeben hat, haben sie jedenfalls verloren. Gegenüber dieser Parallelwelt obliegt es den Mitgliedern der internationalen Staatengemeinschaft, die Belange des öffentlichen Wohls beständig und konsequent zur Geltung zu bringen.

Insgesamt darf man der mittlerweile entstandenen normativen Ordnung der Welt einen bemerkenswerten Perfektionsgrad bescheinigen. In der theoretischen Diskussion hat dieser Befund zur These von der Konstitutionalisierung des Völkerrechts geführt. ${ }^{*}$ Beim Blick auf die Realitäten der heutigen Weltpolitik ist man indes von einem Zustand der Zufriedenheit weit entfernt. Die Leitprinzipien des Völkerrechts werden zwar in den Vereinten Nationen von breiten Mehrheiten fast einhellig immer wieder beschworen, ${ }^{*}{ }^{6}$ werden aber im Alltag nicht nur gelegentlich mit Füßen getreten. Beispiele lassen sich in jeder Ausgabe einer Tageszeitung finden. Die Kriege im Südsudan wie in Syrien, der Zerfall Libyens wie auch die von außen unterstützten gewaltsamen separatistischen Bewegungen im Osten der Ukraine mögen als Beispiel dienen, hinter denen sich unendliches menschliches Leid verbirgt. Nach wie vor ist die Sklaverei nicht ausgerottet, und Millionen von Frauen werden zur Zwangsprostitution missbraucht, auch und gerade in den jedenfalls technologisch fortgeschrittenen westlichen Staaten. Diese kurze exemplarische Liste ließe sich fast beliebig verlängern.

Hat das Völkerrecht seine Steuerungskraft verloren? Es bedarf keiner langen Begründung für die Aussage, dass kein Rechtssystem perfekt ist. Es soll ja gerade die soziale Wirklichkeit disziplinieren, so dass Abweichungen und Unvollkommenheiten geradezu systemimmanent vorausgesetzt sind. Letzten Endes aber sollte sich die normative Ordnung durchsetzen. Bleiben Verletzungshandlungen generell und ständig folgenlos, so bricht irgendwann das Konzept einer Rechtsordnung zusammen. Was ursprünglich Recht genannt wurde, zerfällt zu bloßer politischer Rhetorik. In einem rechtlosen Zustand gibt es nicht nur Verlierer, sondern auch Gewinner. Es sind durchweg die mächtigen Staaten, die ihren Nutzen aus einer Lage ziehen, wo die Regelhaftigkeit als Relikt der Vergangenheit in die Ecke gestellt wird. Demgegenüber haben vor allem die kleineren und mittleren Staaten ein lebhaftes Interesse an einem Umgang, der auf festen Regeln beruht, wo vor allem das Prinzip der souveränen Gleichheit hochgehalten wird.

Versucht werden soll zunächst, durch einen kursorischen Rückblick auf die jüngere Geschichte herauszufinden, wie sich auf dem Gebiete des Völkerrechts Faktizität und Normativität zueinander verhalten, um auf diese Weise in Erfahrung zu bringen, wo die Gründe für eine Schwäche des Völkerrechts liegen können. Daran anschließend soll eine spezifische Untersuchung der gegenwärtigen Rechtslage aufzeigen, mit welchen besonderen Schwierigkeiten man sich bei einer solchen Analyse in der Gegenwart auseinanderzusetzen hat. Lässt sich der Traum von einer großen Friedensordnung verwirklichen?

4 Vgl. etwa Christian Tomuschat, 'International Law as a Coherent System: Unity or Fragmentation?', in: Mahnoush H. Arsanjani et al. (eds.), Looking to the Future. Essays on International Law in Honor of W. Michael Reisman (Leiden and Boston: Martinus Nijhoff, 2011) 323, 354.

5 Vgl. etwa Anne Peters, 'Konstitutionalismus als globale Errungenschaft', in: Jost Delbrück et al. (eds.), Aus Kiel in die Welt (Berlin: Duncker \& Humblot, 2014) 127-138.

6 Vgl. insbesondere die Declaration of the High-level Meeting of the General Assembly on the Rule of Law at the National and International Levels, GV-Resolution 67/1, 24 September 2012. 


\section{Das Völkerrecht als grenzüberschreitendes Ordnungssystem - Historischer Rückblick}

\section{1) Das europäische Völkerrecht}

Üblicherweise wird als Zeitpunkt für das moderne Völkerrecht der Friedensschluss von Münster und Osnabrück des Jahres 1648 angesetzt. ${ }^{*} 7$ Alternativ oder gleichzeitig wird auch Hugo Grotius als Vater der neuzeitlichen Völkerrechtsordnung genannt. ${ }^{*}$ Offensichtlich ist dies eine typische westliche Sichtweise. Über Jahrhunderte entwickelte sich das Normengerüst, das wir heute Völkerrecht nennen, im gegenseitigen Verkehr der christlichen europäischen Staaten untereinander. Vor allem durch die Forschungen des japanischen Autors Onuma Yasuaki wissen wir heute, dass es auch in Ostasien Regeln für den Verkehr zwischen den Völkern gab, die einen hohen Grad von Komplexität erreichten. ${ }^{*}$ Auch das Verhältnis der europäischen zu den arabischen Staaten war teilweise durch rechtliche Regeln geprägt und verengte sich nicht ausschließlich auf Kampf und Gewalt. Aber die europäischen Staaten bewahrten sich doch stets einen Vorsprung nicht nur durch ihre Eroberungspolitik in anderen Weltteilen, sondern auch durch eine gesteigerte Kommunikationsfähigkeit, die es ihnen erlaubte, die von ihnen geübte Praxis zu konzeptualisieren und als ein allgemein verbindliches Rechtssystem darzustellen. Damit war ein Monopolanspruch erhoben, der allerdings nicht nur auf Arroganz beruhte, sondern seine Ursachen auch in schlichter Unkenntnis hatte. Wenig oder gar nichts wussten die kleineren und mittleren europäischen Staaten von der Praxis der Herrschaftsgebilde in Afrika und Asien, und die Autoren der völkerrechtlichen Traktate waren als Schreibstubengelehrte meist von der Praxis noch weiter entfernt als die den Regierungen als Berater zur Seite stehenden Juristen. Rührend ist es zu lesen, wenn in den Lehrbüchern des Völkerrechts vom Anfang des 19. Jahrhunderts die existierenden Staaten einzeln aufgezählt werden, angefangen vom (alten) Deutschen Reich, Frankreich und Spanien ${ }^{* 10}$ bis hin zur Republik San Marino. ${ }^{*} 11$ Der hier zur Schau getragene Provinzialismus war kein Anzeichen dominanter Selbstsicherheit, sondern erwuchs eher einem Gebot weiser Selbstbeschränkung, da über diesen begrenzten territorialen Kreis hinaus die eigene Lebenserfahrung nicht reichte und demgemäß auch keine Aussagen über die dabei geübte Praxis gemacht werden konnten.

Erste entscheidende Schritte hin zu einer weltumspannenden Ordnung machte das Völkerrecht europäischer Prägung erst, als im Jahre 1856 die Türkei zu den „Vorteilen“ dieses Rechtssystems „zugelassen“ wurde. ${ }^{*}{ }^{12}$ An der Gründung des Völkerbundes im Jahre 1920 waren ursprünglich 32 Staaten beteiligt, unter ihnen 4 asiatische, 2 afrikanische (Liberia, Südafrika) und 9 Staaten aus Lateinamerika. Immer noch standen aber weite Teile Asiens und Afrikas unter Kolonialherrschaft. Im Wesentlichen war der Völkerbund von den europäischen Hauptmächten dominiert, insofern die außereuropäischen Staaten noch in einer Minderheitsposition verblieben. Erst die Charta der Vereinten Nationen setzte mit ihrer Proklamation des Selbstbestimmungsrechts der Völker dieser Differenzierung ein Ende, wenn sie zunächst auch nicht den Mut hatte, die Kolonialherrschaft für überwunden zu erklären. In Art. 73, der „Erklärung über Hoheitsgebiete ohne Selbstregierung“, wurde die Verpflichtung übernommen, die „Selbstregierung“ der betroffenen Völker zu entwickeln. Der Anerkennung eines Rechts auf souveräne Unabhängigkeit kam diese Erklärung nicht gleich. Die Kolonialmächte Frankreich und Großbritannien wollten nach eigenem Ermessen die Art und Weise wie auch die Geschwindigkeit des Emanzipationsprozesses bestimmen. Es bedurfte erst der Erklärung der UN-Generalversammlung vom 14. Dezember 1960 über „Colonial Countries and Peoples“, ${ }^{*} 3$ um der Unabhängigkeitsbewegung zu schnellerer Gangart zu verhelfen. Mit der Anerkennung Südafrikas als eines vom System der Apartheid befreiten demokratischen Mitgliedstaats im Jahre 1994 war dann die

7 Vgl. etwa Wolfgang Preiser, Stichwort ,'History of the Law of Nations, Ancient Times to 1648', in: Encyclopedia of Public International Law, Vol. II (Amsterdam et al.: Elsevier, 1995) 722, 745.

8 Vgl. etwa George Frédéric de Martens, Précis du droit des gens moderne de l’Europe (Göttingen, 1801) 17; Arthur Nussbaum, Geschichte des Völkerrechts (München / Berlin : C.H. Beck, 1960) 126; Preiser (Fn. 7) 744.

9 Onuma Yasuaki, A Transcivilizational Perspective on International Law (Leiden and Boston: Martinus Nijhoff, 2010).

10 Vgl. Martens (Fn. 8) 46.

11 Vgl. Johann Ludwig Klüber, Europäisches Völkerrecht, Bd. 1 (Stuttgart: Cotta, 1821) 60.

12 Pariser Frieden über die Beendigung des Krimkrieges, 30.3.1856, abgedruckt bei: Wilhelm G. Grewe, Fontes Historiae Gentium, Band 3/1 (Berlin / New York: Walter de Gruyter, 1992) 19, Art. 7.

13 GV-Resolution 1514 (XV). 
Kolonialepoche im Wesentlichen abgeschlossen. ${ }^{*}{ }^{*}$ Es bleibt nur noch als Kernproblem die Durchsetzung des Selbstbestimmungsrechts des palästinensischen Volkes durch die Gründung eines souveränen Staates Palästina unter Wahrung der israelischen Sicherheitsinteressen.

Erst von diesem Zeitpunkt an konnte der Gedanke reifen, dass das Völkerrecht eine umfassende Weltordnung für alle Völker der Welt bilden sollte. Die kolonialen Großmächte mochten auch zuvor die rechtliche Möglichkeit gehabt haben, Bindungen für alle Völker unter ihrer Jurisdiktion herzustellen. Aber echte Legitimität konnten solche Rechtsbindungen im Zeichen des aufkommenden und von der UN-Charta auch implizit anerkannten demokratischen Prinzips nicht mehr entfalten. Eine verbindliche Weltordnung muss von den Menschen aller Völker getragen werden. Die Ausübung hoheitlicher Gewalt setzt nach heute allgemein anerkannten Grundsätzen voraus, dass die Gewaltunterworfenen an der Konstituierung und Ausübung solcher Gewalt beteiligt sind. Demokratische Mitbestimmung der Bürger ist kein Luxus, sondern eine notwendige Vorbedingung für legitime Herrschaft.

\section{2) Vom europäischen zum weltumspannenden Völkerrecht}

Damit sind heute alle konzeptionellen Voraussetzungen erfüllt, um jedenfalls den Versuch zu unternehmen, ein Herrschaftssystem mit weltweitem Geltungsanspruch zu errichten, welches die angestrebten Menschheitsziele, wie sie in der UN-Charta angelegt sind, zu verwirklichen. Leicht ist zu erkennen, dass in früheren Jahrhunderten auch bei dem feierlichen Abschluss multilateraler Verträge die Vertragsparteien nicht den Ehrgeiz haben konnten, eine umfassende Friedensordnung zu schaffen, auch wenn die Einleitungsartikel manchmal hehre Ziele verkündeten. So verlangt jeweils Art. 1 der westfälischen Friedensverträge von Münster und Osnabrück von 1648 die Herstellung eines christlichen allgemeinen und immerwährenden Friedens sowie wahre und aufrichtige Freundschaft (Pax Christiana, universalis et perpetua veraque et sincera amicitia), ${ }^{*} 15$ doch konnte es zu der damaligen Zeit nicht gelingen, auch gleichzeitig feste institutionelle Grundlagen für die Sicherstellung dieser Ziele zu schaffen. Eine allgemeine Amnestieklausel (jeweils Art. 2) sollte die Spannungsursachen der Vergangenheit entschärfen und opferte dabei den Gedanken perfekter Gerechtigkeit dem praktischen Bedürfnis, den Boden für ein künftiges friedliches Zusammenleben zu bereiten. Alle „mit Worten, Schriften oder Taten zugefügten Beleidigungen, Gewalttaten, feindselige Handlungen" sollten "gänzlich gegeneinander aufgehoben ... und immerwährendem Vergessen anheimgegeben" sein. In den Artikeln 5, 6 und 7 des Friedens von Osnabrück wurden weitreichende Bestimmungen zur Friedenssicherung getroffen. Einseitige Gewaltanwendung war verboten, und die Vertragspartner wurden sogar aufgefordert, im Falle eines Friedensbruches dem Verletzten militärische Hilfe zu leisten. ${ }^{* 16}$ Bei alldem handelte es sich um Appelle an die Vertragspartner, ganz sicher von den besten Absichten getragen, aber eben doch ohne feste institutionelle Gewährleistung dieser Gebote, auch wenn sie als Reichsgrundgesetze verankert werden sollten. Immerhin war damit im Herzen von Europa ein Friedensbündnis entstanden, das seine Kraft vor allem der Erinnerung an die Schrecken des soeben beendeten Konfliktes verdankte. Insgesamt besaß der Westfälische Frieden Modellcharakter für den ausgewogenen Ausgleich nach einem mörderischen Konflikt. Es gab keine trennscharfe Scheidung zwischen Siegern und Besiegten. Verfestigt wurde nur der Einfluss Frankreichs und Schwedens auf die innerdeutschen Verhältnisse. Als Parteien der Verträge konnten sie als Garantiemächte jederzeit angerufen werden.

Mehr als 150 Jahre später besiegelte der Wiener Frieden von 1815 nach dem Ende der napoleonischen Aggressionen erneut einen Friedenszustand, der von allen Beteiligten nach langen Jahren militärischer Auseinandersetzungen herbeigesehnt wurde. ${ }^{*}{ }^{17}$ Dieser Friedensschluss war von äußerster Nüchternheit und Prägnanz. Die Vertragsparteien verzichteten auf weitreichende Versprechungen, das Herzstück des Wiener Friedens bildeten territoriale Verfügungen, die für die gesamte weitere Dauer des 19. Jahrhunderts bestimmend blieben. Auf die Erarbeitung großartiger Pläne für die Zukunft wurde verzichtet. Lediglich der Vertrag

14 Vgl. die Rede von Präsident Mandela am 3. Oktober 1994 vor der Generalversammlung der Vereinten Nationen, http:// db.nelsonmandela.org/speeches/pub_view.asp?pg=item\&ItemID=NMS204\&txtstr=united\%20nations.

15 Abgedruckt bei Wilhelm G. Grewe, Fontes Historiae Iuris Gentium, Bd. 2 (Berlin / New York: Walter de Gruyter, 1988) 183 und 188.

16 Dieses Instrumentarium war freilich nie zur Anwendung gekommen, vgl. Nussbaum (Fn. 8) 130.

17 Wiener Kongress-Akte, abgedruckt bei Grewe (Fn. 12) 3. 
zwischen den vier Großmächten Österreich, Großbritannien, Preußen und Russland, dem Frankreich später beitrat („Heilige Allianz“), ${ }^{*} 8$ verkündete Frieden als überragendes Ziel, verwob dieses aber aufs Engste mit der Aufrechterhaltung der monarchischen Legitimität und institutionalisierte damit eine Scheidung zwischen den Großmächten auf der einen und den Mittelmächten und Kleinstaaten auf der anderen Seite. ${ }^{*}{ }^{19}$

Das 19. Jahrhundert war insgesamt ein Zeitalter der Nationalstaaten. Sie nahmen als souveräne Einzelakteure die Führungsrolle in internationalen Angelegenheiten für sich in Anspruch, wobei in Deutschland der neu gegründete Deutsche Bund der eigenstaatlichen Entscheidungsfreiheit mit Preußen und Österreich als Führungsmächten nur leichte Fesseln anlegte. Das Völkerrecht war nach wie vor inhaltlich eng begrenzt. Zu seinen Schwerpunkten gehörten territoriale Fragen, das Kriegsrecht sowie vor allem diplomatische und konsularische Beziehungen. Hier stand durchweg die Bilateralität der Rechtsbeziehungen im Vordergrund, wo Rechtstreue durch das Prinzip der Gegenseitigkeit erzwungen wurde. Allerdings entstanden auf technischem Gebiet erste Verwaltungsunionen. Die Schwäche des Völkerrechts war gleichzeitig seine Stärke. Die Staaten wurden nicht durch schwer zu erfüllende Anforderungen überlastet. Ihre Innenpolitik konnten sie fast zur Gänze in eigener Zuständigkeit ohne von außen kommende Vorgaben bestimmen. Einen Höhepunkt der mit großem Einsatz betriebenen Kolonialpolitik war die im Jahre 1885 beschlossene Generalakte der Kongo-Konferenz, die Afrika als ein bloßes Beuteobjekt behandelte. ${ }^{{ }^{20}}$

Nach dem Ende des I. Weltkriegs sollten der Versailler Vertrag mit Deutschland ${ }^{* 21}$ und die übrigen Pariser Vorortverträge ${ }^{* 22}$ mit den anderen besiegten Feindmächten die Grundlage für dauerhaften Frieden und Sicherheit in Europa legen. Diese Verträge selbst beschränkten sich durchweg auf die Ziehung neuer Grenzlinien, während die Neuordnung Europas und der Welt von der Satzung des Völkerbundes ausgehen sollte, die einen Bestandteil des Versailler Vertrages bildete. ${ }^{* 23}$ Hier erhob sich die Präambel zu ehrgeizigen Formulierungen, dass es „zur Förderung der Zusammenarbeit unter den Nationen und zur Gewährleistung des internationalen Friedens und der internationalen Sicherheit“ wesentlich sei, bestimmte grundlegende Verpflichtungen zu erfüllen, insbesondere „nicht zum Kriege zu schreiten“. In Art. 10 wurde dieses Gebot, die territoriale Unversehrtheit und die politische Unabhängigkeit aller Bundesmitglieder zu achten, ausdrücklich als Rechtsverpflichtung statuiert und im Anschluss daran eine Gewährleistung durch den Rat des Völkerbundes ausgesprochen (Art. 11). Bekanntlich ist dieses Ordnungsmodell gescheitert. Es ist Sache der Historiker, ein Urteil darüber abzugeben, welche Gründe für den Misserfolg maßgebend waren. Unter den Gründen lassen sich freilich mehrere klar benennen. Grundlegend waren der Mangel an übereinstimmenden Wertvorstellungen, vor allem nach dem Auftreten der Sowjetunion auf der weltpolitischen Bühne, dann die Diskriminierung des Deutschen Reiches, dem anfänglich ein gleichberechtigter Status verwehrt wurde, ferner die Abwesenheit der USA, deren Senat sich davor scheute, durch eine Ratifizierung der Satzung die USA in die Stellung einer der hauptverantwortlichen Mächte für den Frieden in der Welt zu erheben. Deutlich ist zu erkennen, dass die Mitglieder des Völkerbundes seinerzeit weit davon entfernt waren, eine gemeinsame Konzeption für eine kohärente Weltpolitik zu entwickeln. Im Laufe der wenigen Jahre der Existenz des Völkerbundes zerfiel der ursprüngliche schwache Konsens im Übrigen immer weiter. Im Jahre 1931 drangen japanische Truppenverbände in die Mandschurei ein, 1935 überfiel Italien das Mitgliedsland Abessinien, ohne dass das vorhandene Sanktionspotential den Rechtsbruch verhindern konnte. Eine weitere Schwächung des Völkerbundes wurde durch den Austritt des nationalsozialistischen Deutschen Reiches aus der Organisation im Jahre 1933 bewirkt. So war der Völkerbund auch nicht in der Lage, in den Jahren 1939 und 1940 der Aggression der Sowjetunion gegen Finnland und die baltischen Staaten entgegenzutreten. Auch der spanische Bürgerkrieg lief völlig ohne Beteiligung des Völkerbundes ab, da Spanien kein Mitglied der Weltorganisation geworden war. Im Rückblick erscheint als Hauptmangel des Völkerbundes, dass er den Ausbruch des II. Weltkrieges nicht verhindern konnte.

Trotz dieser Fehlschläge - und gerade wegen dieser Fehlschläge - ließen sich die Siegermächte des II. Weltkrieges nicht davon abschrecken, noch vor dem Ende des Kampfgeschehens Pläne für die Schaffung einer neuen Weltorganisation zu entwerfen. Die UN-Charta wurde in San Francisco am 26. Juni 1945 zu

18 Vom 20.11.1815, ibid., 100.

19 Dazu Hermann Mosler, Die Großmachtstellung im Völkerrecht (Heidelberg: Lambert Schneider, 1949).

20 Vom 26.2.1885, abgedruckt bei Wilhelm G. Grewe, Fontes Historiae Juris Gentium, Band 3/1 (Berlin / New York: Walter de Gruyter, 1992) 297.

21 Abgedruckt bei Grewe, ibid., Band 3/2 (1992) 683.

22 Verträge von St. Germain (Österreich), Neuilly (Bulgarien), Trianon (Ungarn), Sèvres (Türkei), ibid., 701-718.

23 Ibid., 810. 
einer Zeit angenommen, als der Krieg mit Japan noch nicht beendet war. Hellsichtig erkannte man, dass in der Tat das Kriegsverbot der Völkerbunds-Satzung beibehalten und verstärkt werden müsse und dass es vor allem erforderlich sei, ihm - in Gestalt des Sicherheitsrats - eine feste institutionelle Stütze zu geben. Die seitdem vergangenen mehr als 70 Jahre haben gezeigt, dass auch die UN-Charta nicht zu einer definitiven Lösung des Grundproblems der internationalen Staatengemeinschaft, der Gewaltanwendung in den internationalen Beziehungen, geführt hat. Vor allem ist der Sicherheitsrat vielfach nicht bereit, seine Verantwortung zu übernehmen, weil die ständigen Mitglieder ihr Vetorecht zu eigenständigen Machtspielen nutzen, die nicht den Zielen der internationalen Gemeinschaft nützen. Man darf also in der Gegenwart kein optimistisches Fazit ziehen. Trotz ihrer juristischen Perfektion hat es die UN-Charta nicht vermocht, jenen Zustand des friedlichen Ausgleichs herbeizuführen, den sich ihre Verfasser im Jahre des Neuanfangs 1945 erhofft hatten.

\section{Hauptprobleme der Gegenwart \\ 1) Die Moralisierung des Völkerrechts als Gewinn und Risikofaktor zugleich}

Löst man sich von dem Zentralproblem Frieden und Sicherheit, so stößt man bei einer Querschnittsdiagnose auch auf andere Grundprobleme, welche die Diskrepanz zwischen Anspruch und Wirklichkeit in ein grelles Licht rücken. Es ist paradoxerweise die soeben schon beschriebene ethische Anreicherung des Völkerrechts, die an seine Leistungskraft rührt. Zu Recht hat man sich nach den Erfahrungen zweier Weltkriege der Aufgabe gestellt, dem Völkerrecht einen festen moralischen Boden einzuziehen und es nicht nur als technische Apparatur zu betrachten, die zur Verfolgung beliebiger Ziele eingesetzt werden kann. Das Gewaltverbot der Charta wurde mit den vier Genfer Abkommen aus dem Jahre 1949 in das jus in bello hinein grundlegend reformiert und gestärkt. Als weitere Kernelemente des neuen Völkerrechts der Epoche nach dem II. Weltkrieg können die Bestimmungen gelten, dass Verträge, die unter Zwangsanwendung zustande gekommen sind, nicht als rechtsgültig anerkannt werden (Art. 52 der Wiener Vertragsrechtskonvention, WVK) und dass jeder Verstoß gegen jus cogens, die Kernsubstanz des Völkerrechts, einen Vertrag nichtig macht (Art. 53 WVK). Niemals hat man sich indes der Illusion hingegeben, dass die Gewährleistung solcher rechtsethischer Standards ein Kinderspiel wäre. Wenn aber das Völkerrecht als Verkörperung einer gerechten Weltordnung seine Legitimität behalten sollte, so war der Schritt hinüber zu einer neuen Begründung trotz der damit verbundenen Risiken unausweichlich.

Richtig haben die Verfasser der UN-Charta sich nach den Erfahrungen mit dem nationalsozialistischen Deutschland vor allem auch der Frage gestellt, wie verhindert werden kann, dass aus Unruhe, Chaos und Gewaltsamkeit im Inneren eines Landes nachteilige Konsequenzen für den internationalen Frieden erwachsen. In Art. 1 Abs. 3 der Charta ist diese Abhängigkeit des internationalen Friedens von einem Zustand des Friedens im internen Raum der Staaten richtungweisend zum Ausdruck gebracht worden. Hiervon ist ein allgemeiner Anstoß ausgegangen, vom Völkerrecht aus auf das interne Leben der Völker Einfluss zu nehmen. Diese Kehrtwendung macht sowohl die Stärke wie auch die Schwäche des heutigen Völkerrechts aus. Seine Stärke ist, dass es sich als moralisch geschlossenes Gebilde darstellt und dabei die frühere ausschließliche Ausrichtung auf die zwischenstaatlichen Beziehungen abgelegt hat. Auf der anderen Seite hat es mit dem Vordringen in den innerstaatlichen Raum eine Aufgabe angepackt, für deren Bewältigung ihm durchweg nur unzureichende Handlungsmittel zur Verfügung stehen.

Wenn dem Völkerrecht im Zuge dieser Neuorientierung auch die Aufgabe zugewiesen wird, die materiellen Grundbedürfnisse des Menschen zu befriedigen, und eine fürsorgerische Sozialpolitik zu betreiben, so greift es damit tief in die Entscheidungsfreiheit der Staaten ein. Im Grunde manifestiert sich hier eine völlige Abkehr vom Völkerrecht klassischer Prägung, für das die Trennung von Innen und Außen zu den Grundpfeilern des systematischen Verständnisses gehörte. In seiner einflussreichen Schrift „Völkerrecht und Landesrecht“ hat im Jahre 1899 Heinrich Triepel diese Scheidung dogmatisch untermauert. ${ }^{* 2}$ Für ihn waren Völkerrecht und innerstaatliches Recht dem Grundsatz nach unterschieden durch den Adressatenkreis und vor allem die normativen Inhalte. Insbesondere die Menschenrechte waren für ihn entsprechend

24 Leipzig: Hirschfeld. 
der damals vorherrschenden Konzeption kein denkbarer Gegenstand des Völkerrechts, und diese Konzeption hat bis in die neuere Zeit hinein ihren Niederschlag gefunden in Art. 2 Abs. 7 der UN-Charta, wonach es einen Bereich der inneren Angelegenheiten gibt, in den von den Organen der Vereinten Nationen nicht eingegriffen werden darf. Nach einer vor allem von den sozialistischen Staaten über Jahrzehnte mit großer Vehemenz vertretenen Auffassung gehörte die tatsächliche Praxis der Menschenrechte zu diesem geschützten Bereich, der auch durch das Interventionsverbot des allgemeinen zwischenstaatlichen Völkerrechts gegen Einflussnahme von außen ${ }^{*} 25$ rechtlich abgeriegelt sein sollte. Mit der Annahme der beiden Weltmenschenrechtspakte im Jahre 1966 und dem Beginn der Tätigkeit des Menschenrechtsauschusses zur Prüfung von Berichten der Staaten über ihre Praxis im Jahre 1977 hat dieses Theoriemodell seine Grundlagen eingebüßt. So haben die Menschenrechte die klassische Trennmauer zwischen Völkerrecht und innerstaatlichem Recht fast gänzlich niedergelegt. Das innere Leben des Staates ist rechtlich in den Status der Transparenz erhoben worden. Der Staat schuldet der internationalen Gemeinschaft Rechenschaft im Hinblick auf jede Maßnahme, die in irgendeiner Weise Menschenrechte berührt - und das betrifft nahezu das gesamte Staatshandeln. Gefordert wird im Grunde good governance als Rechtsgebot.

Aber die Schwierigkeiten, diesem Anspruch gerecht zu werden, sind enorm. Das Völkerrecht entwickelt sich gleichsam zum Legitimitätsmaßstab, der neben die von den Völkern in eigener demokratischer Verantwortung beschlossenen Legitimitätskriterien tritt. Dies muss deshalb jedenfalls langfristig zu Spannungen führen, weil die völkerrechtlichen Instrumente nicht nur erfreulich stabil und fest, sondern gleichzeitig auch beklagenswert starr sein können. In einem demokratischen Gemeinwesen gehört grundsätzlich auch die Überprüfung des gegebenen Normenbestandes zu den selbstverständlichen und jederzeit verfügbaren Optionen. ${ }^{{ }^{2} 6}$ Vor allem bei den menschenrechtlichen Schutzinstrumenten sind Veränderungen durchweg nur im Wege des breiten Konsenses denkbar, und die gefestigte richterliche Rechtsprechung einer internationalen Spruchinstanz lässt sich kaum aus den Angeln heben. Richter selbst haben die Neigung, ihre eigenen Judikate zu dogmatisieren und sie für sakrosankt zu halten.

\section{2) Stabilisierende Elemente}

Nicht zu übersehen sind andererseits die Signale, die dem Völkerrecht konstruktive Stabilität verleihen. In der heutigen Zeit lassen sich seine Schwächen und Stärken besser erkennen als etwa vor rund 100 Jahren in Paris nach dem Ende des I. Weltkrieges, als man noch in den klassischen Denkmustern der Zwischenstaatlichkeit verhaftet war.

Als wichtiger Pluspunkt ist zunächst die Tatsache zu vermerken, dass alle Staaten der Welt bereit sind, die Existenz des Völkerrechts und seine Geltungskraft anzuerkennen. Jedenfalls auf der politischen Ebene der Politiker und Diplomaten gibt es keine einzige Stimme, welche das Völkerrecht als Regulierungsinstrument für die internationalen Beziehungen grundsätzlich ablehnen würde. ${ }^{* 27}$ Dies gilt zunächst für das Handwerkszeug des völkerrechtlichen Vertrages, der von allen Regierungen als Handlungsmittel genutzt wird. Seine Nützlichkeit und Unersetzbarkeit ist so offensichtlich, dass es dazu keiner langen Diskussion bedarf - die auch nie geführt worden ist. Verträge sind ihrer Natur nach, da sie auf dem Konsens der Vertragsparteien aufbauen, Instrumente des friedlichen Ausgleichs. Nur wer - unter Beseitigung des Prinzips der souveränen Gleichheit - der Monopolherrschaft eines einzigen Landes das Wort reden wollte, könnte das autoritäre Diktat als Ersatz für den Vertrag anbieten. Was für den Vertrag selbst gilt, trifft auch für seine Anwendungsmodalitäten zu. Das Vertragsregime, wie es in der Wiener Vertragsrechtskonvention seinen Niederschlag gefunden hat, steht im Grundsatz unangefochten da, auch wenn über Details - fast zwangsläufig - gestritten wird. Damit hat das Völkerrecht feste handwerkliche Grundlagen, die ihm auch in Zukunft erhalten bleiben werden. Dies ist umso wichtiger, als ja die große Mehrzahl der völkerrechtlichen Verpflichtungen aus dem Abschluss völkerrechtlicher Verträge erwächst. Der völkerrechtliche Vertrag ist das Arbeitspferd der internationalen Beziehungen.

25 Declaration on Principles of International Law concerning Friendly Relations and Co-operation among States in accordance with the Charter of the United Nations, GV-Resolution 2625 (XXV), 24. Oktober 1970, Prinzip 3.

26 Dazu Isabelle Ley, Opposition im Völkerrecht (Heidelberg et al.: Springer, 2014) 201.

27 Die These von Jack L. Goldsmith und Eric A. Posner, The Limits of International Law (Oxford: Oxford University Press, 2010), dass die Staaten das Völkerrecht nur als eine Sammlung von unverbindlichen Strategieregeln betrachten würden, ist weit überspitzt. 
Völkerrechtliche Verträge sind auch die Grundlage für die heute existierenden Internationalen Organisationen. Was dort niedergelegt ist, hat dank des im Vertragsrecht geltenden Konsensprinzips eine erhöhte Bestandsgarantie. Vor allem die Großmächte, die auf Grund der im Jahre 1945 herrschenden Machtlage für sich einen ständigen Sitz im UN-Sicherheitsrat erringen konnten, wären in einer offensichtlichen Zwangslage, wenn sie die Bindungskraft des Völkerrechts generell bestreiten wollten. Denn damit stellen sie unvermeidlich auch ihre eigene Machtstellung in der Weltorganisation in Frage. Diese Privilegierung stellt für sie ein kostbares Gut dar, das sie bei einer Revision der Charta in keinem Falle wieder in vollem Umfang erhalten würden, was vor allem für die europäischen Mittelmächte Frankreich und Vereinigtes Königreich gilt. Jedenfalls die ständigen Mitglieder des Sicherheitsrates darf man deswegen zu den unbeugsamen Befürwortern des Völkerrechts in seiner heutigen Gestalt rechnen. ${ }^{*}{ }^{28}$

$\mathrm{Zu}$ den Neuerungen mit einer jedenfalls auf lange Sicht erheblichen strukturellen Wirkung darf man wohl auch die Tatsache rechnen, dass mittlerweile der Grundsatz sich definitiv Bahn gebrochen hat, wonach jeglicher im Sinne des Völkerrechts rechtswidrige Akt die Verantwortlichkeit des handelnden Staates nach sich zieht. Die Erarbeitung der Vorschriften über „Responsibility of States for internationally wrongful acts" durch die Völkerrechtskommission der Vereinten Nationen im Jahre 2001 und ihre Kenntnisnahme durch die Generalversammlung am 12. Dezember $2001^{* 29}$ ist überwiegend als ein bloß rechtstechnischer Vorgang verstanden worden. Gesagt wurde im Allgemeinen, dass es sich hier lediglich um die Kodifikation von ohnehin geltendem Gewohnheitsrecht handele. ${ }^{*}{ }^{30}$ Schon dies ist zweifelhaft, da die Völkerrechtskommission zu einem beträchtlichen Anteil rechtschöpferisch gehandelt hat. ${ }^{*}{ }^{11}$ Verschiedene Artikel des Regelentwurfs sind neu und lassen sich nur schwer auf eine bereits bestehende Rechtsquelle zurückführen. Jedenfalls aber ist der Entwurf ein Bekenntnis zu der Bindungskraft des Völkerrechts, wenn es dort in Artikel 1 heißt:

Every internationally wrongful act of a State entails the international responsibility of that State.

Es lässt sich gar nicht leugnen, dass dieser Satz eher aus dem Himmel der Theorie stammt statt aus der Schmiedewerkstatt der Praxis. Es tragen sich alltäglich Hunderte oder sogar Tausende von rechtswidrigen Handlungen zu, die keinerlei Weiterungen nach sich ziehen, vor allem weil der Geschädigte es nicht für angemessen hält, Wiedergutmachungsforderungen zu stellen. Dennoch hat die zitierte Aussage ihre Bedeutung, indem sie mit allgemeiner Billigung in der internationalen Gemeinschaft klarstellt, dass Völkerrecht eine spezifische Bindungskraft besitzt, deren Verletzung die in dem Entwurf näher umschriebenen Wirkungen nach sich zieht. Damit ist eine Antriebskraft für die Einhaltung völkerrechtlicher Verpflichtungen benannt, auf die sich jedes von einer Rechtsverletzung betroffene Völkerrechtssubjekt berufen kann. Das allgemeine Völkerrecht bildet also bei normativer Betrachtung eine der festesten Grundlagen für die Einhaltung des Völkerrechts insgesamt.

Ein hohes Maß an Effektivität darf man auch der Mehrzahl der eher unpolitischen technischen Regelungen des Völkerrechts attestieren. Die WTO hat sich zu einem Verhaltensmaßstab für den internationalen Handelsverkehr entwickelt, der durch die bestehenden Beschwerdemöglichkeiten wirksam abgestützt wird. Zu den positiven Aspekten gehören insgesamt die Arbeit der Sonderorganisationen der Vereinten Nationen sowie die weltweit funktionierenden Regime zum Schutz der Gemeingüter der Menschheit. Hier, wo es weniger um die Verteilung der erzeugter Güter geht als um die Sicherung der Lebenschancen der Menschheit insgesamt, müsste sich nach rationalen Maßstäben letzten Endes die Vernunft durchsetzen, welche Erhaltung und Schutz propagiert. Für das Rechtsregime der Weltmeere hat die Staatengemeinschaft in der Seerechtskonvention vom 10. Dezember 1982 eine sorgfältig austarierte Kompromisslösung gefunden, die gewiss nicht jede kontroverse Einzelheit geklärt hat, aber doch einen

28 Zur ambivalenten Haltung Russlands s. Lauri Mälksoo, Russian Approaches to International Law (Oxford: Oxford University Press, 2015). Eine Durchsicht des Chinese Journal of International Law ergibt, dass dort höchste Regierungsmitglieder sich durchweg für die Einhaltung des Völkerrechts in seiner heutigen Gestalt einsetzen, vgl. etwa Deming Huang, Yuan Kong und Hua Zhang, 'Symposium on China's Peaceful Development and International Law', 5 Chinese Journal of International Law (2006) 261, 262.

29 GV-Resolution 56/83, 12.12.2001.

30 James Crawford, 'State Responsibility', in: Rüdiger Wolfrum (ed.), Max Planck Encyclopedia of Public International Law, Vol. IX (Oxford: Oxford University Press, 2012) 517, 532, nennt die Artikel des ILC-Entwurfs "part of the fabric of general international law".

31 Vgl. insbesondere die Regeln über Gegenmaßnahmen, dazu James Crawford, The International Law Commission's Articles on State Responsibility (Cambridge: Cambridge University Press, 2002) 47-56. 
Fundus bildet, der im Gesamtpaket als ein Muster für sorgfältige Abwägung aller vorhandenen Interessen gelten kann. Niemand kann dem Regime der Seerechtskonvention Einseitigkeit oder versteckte Parteinahme vorwerfen. Natürlich sind auch hier Anpassungen und Weiterentwicklungen notwendig. So war das immense Ausmaß der Meeresverschmutzung durch Eintrag von festen oder gasförmigen Abfällen während der Dauer der Seerechtskonferenz noch nicht mit ausreichender Schärfe erkannt worden. Hier bedarf es zusätzlicher Regelungen, die nicht leicht auf den Weg zu bringen sein werden. Aber es besteht Hoffnung, weil sich mittlerweile gut eingespielte Verhandlungsmechanismen herausgebildet haben. Einen weiteren Grund für Optimismus bildet das im Seerecht stark ausgebildete gerichtliche Streitbeilegungssystem, das einige Erfolge für sich verbuchen kann, das aber auch mit der chinesischen Weigerung, den Schiedsspruch zu den Rechtsverhältnissen im südchinesischen $\operatorname{Meer}^{*} 32$ zu respektieren, einen schweren Rückschlag erlitten hat.

Insgesamt darf man wohl davon ausgehen, dass bei der Verwaltung gemeinsamer Güter der Menschheit zu den bereits erzielten Erfolgen noch weitere hinzutreten werden. Offensichtlich haben sich die Staaten nicht der Einsicht verschlossen, dass ein Verbot von Stoffen, die zum Abbau der Ozonschicht führen, im Interesse aller liegt. Das zu diesem Zweck erarbeitete Protokoll von Montreal vom 16. September 1987 zählt heute nicht weniger als 197 Vertragsparteien. Im Vertrag von Paris vom 12. Dezember $2015^{*} 33$ haben sich die Parteien geeinigt, auf die Ziele des Klimaschutzes zumindest hinzuarbeiten, auch wenn die Verpflichtungen mit äußerster Flexibilität formuliert sind.

Betrachtet man das Panorama des Völkerrechts von einer höheren Warte aus, so sticht hervor, dass das moderne Völkerrecht über eine Vielzahl von Verfahren verfügt, in denen streitige Probleme geklärt werden können. Dies gilt für alle Lebensbereiche. Zwar ist die Einschaltung des Internationalen Gerichtshofs nach wie vor in das Ermessen der Streitpartien gestellt. Bei der Streiterledigung gehört der Gang nach Den Haag lediglich zu einer der möglichen Optionen. Indes bieten sich vor allem die internationalen Organisationen als Gesprächsforen an, wo die zuständigen Gremien zugleich wichtige Vermittlungsfunktionen ausüben können. Auf weltweiter Ebene steht es jedem Mitgliedstaat jederzeit frei, die Dienste des UN-Generalsekretärs oder sonstiger zuständiger Sonderorgane in Anspruch zu nehmen, wenn bilaterale Gespräche ihre Ergebnislosigkeit gezeigt haben. Sobald es um Probleme von Krieg und Frieden geht, kommt es heute fast automatisch zur Einschaltung des Sicherheitsrates und/oder seiner Mitglieder. Undenkbar wäre heute, dass Mächte in ähnlicher Weise unbewusst in einen kriegerischen Konflikt hineinschlittern, wie dies nach der Deutung des Oxforder Historikers Christopher Clark im Jahre 1914 geschehen ist, ${ }^{*}{ }^{4}$ nicht zuletzt deswegen, weil die Regierungsführer der Großmächte keine institutionellen Kontakte zueinander besaßen und ihre Entscheidungen auf engster Wissensbasis ohne angemessene Beratung in einer Atmosphäre der Vereinzelung trafen. Vermittlungs- und Ausgleichsmechanismen werden heute nicht nur von den Vereinten Nationen, sondern in reicher Vielzahl auch auf regionaler Ebene angeboten, in Europa etwa im Rahmen des Europarates, der Europäischen Union und der OSZE.

$\mathrm{Zu}$ den klassischen Verfahren diplomatischer Prägung treten heute vielfach förmliche Verfahren gerichtlicher Art hinzu, die vor allem auf dem Gebiet der Menschenrechte einen ungeheuren Aufschwung genommen haben. Bekannt ist die Führungsrolle, die seit vielen Jahrzehnten der Europäische Menschenrechtsgerichtshof in Straßburg Zug um Zug aufgebaut hat, so dass er als Vorbild für den Interamerikanischen Menschenrechtsgerichtshof wie auch für den Afrikanischen Gerichtshof für Menschenrechte und die Rechte der Völker dient. Der Straßburger Gerichtshof, der im Jahre 2016 wieder eine Rekordzahl von Fällen erledigt hat, ${ }^{*}{ }^{35}$ hat die Zuständigkeit, das gesamte Handeln aller 47 Vertragsparteien der Europäischen Menschenrechtskonvention zu kontrollieren. Bisher sind seine Entscheidungen meist anstandslos von den beklagten Staaten hingenommen worden, obwohl vielfach die Vollstreckung erst mit Hilfe zäher Bemühungen des Ministerkomitees nach langer Frist gesichert werden konnte. In jüngster Zeit hat sich überdies eine allgemeine Debatte über die Legitimation des Gerichtshofs zur Beurteilung von Problemlagen mit typischer nationaler Färbung entwickelt. In Großbritannien will man seine Rechtsprechung

\footnotetext{
32 Award of 12 July 2016, PCA Case No. 2013-19, Republic of the Philippines v. People's Republic of China, http://www. pcacases.com/web/sendAttach/2086.

33 Der Vertrag hat bereits 136 Parteien und ist am 4. November 2016 in Kraft getreten.

34 Christopher Clark, Die Schlafwandler (München: Deutsche Verlagsanstalt, 2013).

3538.505 Fälle, s. European Court of Human Rights, Annual Report 2016, 193.
} 
zum Wahlrecht verurteilter Straftäter nicht hinnehmen, ${ }^{*}{ }^{36}$ und Russland hat ihm erst jüngst den Fehdehandschuh hingeworden, indem zunächst der Russische Verfassungsgerichtshof in einer Grundsatzentscheidung festgestellt hat, dass Straßburger Entscheidungen gegen die russische Verfassung verstoßen könnten, ${ }^{, 37}$ und indem später dieses höchstrichterliche Dictum durch Gesetz abgesichert wurde. ${ }^{*}{ }^{*} \mathrm{Im}$ Falle des Urteils in der Yukos-Sache, wo der Gerichtshof wegen zahlreicher Unregelmäßigkeiten des Verfahrens eine Wiedergutmachungsleistung in Höhe von 1 Milliarde 866 Millionen Euro angeordnet hat, ${ }^{*} 39$ ist diese Bremsfunktion ohne Umschweife in Anspruch genommen worden. ${ }^{*}{ }^{40}$ Hier zeigen sich Grenzen richterlicher Macht, wenn ein Land den Eindruck gewinnt, dass seine Verfassungsidentität beeinträchtigt worden sei. Auch das deutsche Bundesverfassungsgericht hat prinzipiell den Standpunkt bezogen, dass die Elemente der Verfassungsidentität eine Mauer bildeten, vor der das Völkerrecht Halt machen müsse. ${ }^{*} 41$ Unleugbar ist, dass das Völkerrecht seine Legitimation von den Staaten her bezieht, wo die Legitimität aller öffentlichen Gewalt ihren Ursprung hat.

Als Erfolg hat man bisher die Arbeit der internationalen Strafgerichte bewertet, die ja dazu bestimmt sind, der Wahrung der Kernsubstanz der völkerrechtlichen Ordnung durch strafrechtliche Sanktionen zu dienen. Vor allem die beiden vom Sicherheitsrat eingerichteten internationalen Strafgerichte für das ehemalige Jugoslawien (ICTY) und für Ruanda (ICTR) können in der Tat eine bemerkenswerte Bilanz vorweisen. Weniger günstig ist der Internationale Strafgerichtshof (ICC) zu beurteilen, der ja lediglich auf einem Vertrag beruht, den die Staaten zu ratifizieren frei sind. Keine der Großmächte China, Russland und USA hat sich der Jurisdiktion des ICC unterworfen. Aus Afrika ist die Kritik lautstark geäußert worden, dass sich der ICC im Grunde nur auf Afrika konzentriere und deswegen seine Unparteilichkeit eingebüßt habe. In der Tat hat mittlerweile ein Land (Burundi) seine Ratifikation des Römischen Statuts schon wieder widerrufen, und Südafrika steckt insoweit noch in einem Abwägungsprozess. Eine internationale Strafgerichtsbarkeit kann auf Dauer nur funktionieren, wenn gleiches Recht für alle gilt. Die Zukunftsaussichten des IStGH bleiben daher im Dunkel schwankender Prognosen höchst unsicher.

Als Erfolgsmodell lässt sich der europäische Integrationsprozess bewerten, der seit dem Inkrafttreten des Vertrages über die Europäische Gemeinschaft für Kohle und Stahl im Jahre 1952 bis zum heutigen Tage friedliche Beziehungen unter den Mitgliedstaaten hat gewährleisten können. Obwohl in anderen Weltregionen die heutige Europäische Union sich bisher nicht als wirkmächtiges Vorbild hat durchsetzen können, und obwohl der bevorstehende Austritt des Vereinigten Königreichs die Attraktivität des europäischen Zusammengehens empfindlich schwächen wird, bleibt dieser Solidarverbund eine große Hoffnung echter genossenschaftlicher Zusammenarbeit ohne strukturelle Vorherrschaft einzelner Mitgliedsländer. Fast unvermeidlich wird man sich außerhalb Europas bei ähnlichen Planungen an der europäischen Vorreiterrolle orientieren müssen.

36 Ausgangspunkt war der Fall Hirst v. United Kingdom (No. 2), Beschwerde Nr. 74025/01, 6.10.2005. Bestätigung der Rechtsprechung Hirst in Greens and M.T. v. United KIngdom, Beschwerde Nr. 60041/08, 23.11.2010, und Firth and Others $v$. United Kingdom, Beschwerde Nr. 47784/09, 12.8.2014. See also the judgment of the Russian Constitutional Court of 19 April 2016, Nr. 12-P/2016, on the judgment of the European Court of Human Rights in Anchugov and Gladkov.

37 Russischer Verfassungsgerichtshof, 14.7.2015, teilweise wiedergegeben bei Matthias Hartwig, 'Vom Dialog zum Disput? Verfassungsrecht vs. Europäische Menschenrechtskonvention - Der Fall der Russländischen Föderation', 44 Europäische Grundrechte-Zeitschrift (2017) 1, 5.

38 Gesetz vom 14.12.2015, ibid., 8. S. dazu die negative Stellungnahme der Venedig-Kommission des Europarats, Nr. 832/2015, 13.6.2016, Dok. CDL-AD(2016)016.

39 Yukos v. Russian Federation, Beschwerde Nr. 14902/04, 31.7.2014.

40 Mit Urteil vom 19.1.2017 hat der Russische Verfassungsgerichtshof entschieden, dass das Urteil in Russland nicht vollstreckbar sei: https://acerislaw.com/de/european-court-human-right/.

41 Vgl. insbesondere das Urteil Görgülü des Bundesverfassungsgerichts, 14.10.2004, BVerfGE 111, 307, 319. Das Urteil des italienischen Verfassungsgerichtshofs Nr. 238 vom 22.10.2014, das dem Spruch des Internationalen Gerichtshofs in der Sache Deutschland gegen Italien, Urteil vom 3.2.2012, ICJ Reports 2012, 99, die Verbindlichkeit in Italien verweigerte, muss eher als zufallsbedingter Unfall bezeichnet werden. 


\section{3) Risikobereiche}

Kritische Aspekte enthüllt, wie im Vorstehenden schon angedeutet, der Blick auf andere Materien des Völkerrechts, wo die effektive Durchsetzung seiner Normen ständig in Frage steht. Hier müssen zunächst die Menschenrechte genannt werden, vor allem in ihrer Ausweitung auf die Rechte der „zweiten“ und „dritten“ Generation.

Die klassischen bürgerlichen Rechte gehören seit mehr als zweihundert Jahren zum Kernbestand der westlichen Demokratien, angefangen mit der französischen Déclaration des droits de l'homme et du citoyen und der amerikanischen Bill of Rights, seit der europäischen Renaissance der Jahre 1945 und 1989 in ganz Europa. Alle diese Rechte - Recht auf Leben, auf Freiheit, Meinungsfreiheit, Schutz vor körperlicher Misshandlung - gehörten zunächst zum innerstaatlichen Verfassungsrecht, ehe sie über die Europäische Menschenrechtskonvention, den Internationalen Pakt über bürgerliche und politische Rechte wie auch die anderen regionalen Menschenrechtsinstrumente in das Völkerrecht hineinwuchsen. Im Umgang mit diesen Rechten liegt ein breites Erfahrungsmaterial vor. Durch die Beschwerde zum Straßburger Gerichtshof ist in weitem Umfang sichergestellt, dass aus bloßen papierenen Versprechungen echte Schutzpositionen tatsächlicher Art werden. Aber der juristische Perfektionismus ist heute ersichtlich nicht mehr in der Lage, in Mitgliedsländern, in denen starke strukturelle Defizite herrschen, eine entscheidende Wende zum Besseren herbeizuführen. In Russland wie auch in der Türkei sind de facto die demokratischen Freiheitsrechte abgeschafft. Oppositioneller Widerspruch wird als strafbarer Landesverrat oder Terrorismus abqualifiziert. Die Straßburger Maschine läuft noch, immer noch werden Einzelfälle entschieden, und vielfach leisten die Behörden tatsächlich die den Beschwerdeführern zuerkannten Schadensbeträge. Aber das System ist ins Herz getroffen worden, wenn jeder, der von seinem Recht der Meinungsfreiheit Gebrauch macht, unverzüglich mit strafgerichtlicher Verfolgung rechnen muss. Die Schranke der nationalen Identität wird offensichtlich missbraucht, wenn sie zur Abwehr und Unterdrückung elementarer demokratischer Freiheiten benutzt wird.

Noch größere Schwierigkeiten bereitet eine Beurteilung der Effektivität der wirtschaftlichen, sozialen und kulturellen Rechte, die heute in allen Systemen, die überhaupt einen Menschenrechtsschutz kennen, hinsichtlich ihres materiellen Werts auf die gleiche Stufe wie die klassischen Freiheitsrechte gestellt werden. Es ist gleichsam ein Dogma der Menschenrechtsbewegung geworden, dass keine Unterscheidung zwischen den verschiedenen Gruppen von Rechten getroffen werden dürfe. Die Generalversammlung hat sich wiederholt dezidiert in diesem Sinne geäußert, ${ }^{*} 42$ und Widerspruch macht sich rar.

In einem politisch-moralischen Sinne haben diejenigen, die sich für die Gleichstellung der beiden Fallgruppen einsetzen, völlig Recht. Nahrung, Kleidung, Wohnung und Gesundheit gehören zu den Grundbedürfnissen des Menschen. Sie sind für ihn ebenso wichtig wie die elementaren Freiheitsrechte, ja geradezu existenzentscheidend. ${ }^{*} 43$ Nur ist nicht zu verkennen, dass das Völkerrecht mit seiner Forderung, diese Grundbedürfnisse zu sichern, den Staaten eine Last aufbürdet, der sie häufig gar nicht gerecht werden können, auch wenn sie sich dazu durch förmlichen Vertragsschluss bereit erklärt haben.

Hier gelangt man an einen der neuralgischen Punkte des heutigen Völkerrechts. Das Netz der multilateralen Verträge ist eindrucksvoll. Der Mitgliederbestand umfasst gelegentlich sogar mehr als die 193 Staaten, die Mitglieder der Vereinten Nationen sind. Aber die formale Vertragsmitgliedschaft und die effektive Leistungskraft fallen vielfach weit auseinander. Regierungen sind bereit, Vertragsbindungen zu übernehmen, zu deren Erfüllung sie nicht in der Lage sind oder die sie gar nicht zu erfüllen beabsichtigen. Damit ist die gesamte Logik des völkerrechtlichen Vertrages in Frage gestellt, die davon ausgeht, dass jede Vertragsbindung auf einen staatlichen Akteur zurückzuführen ist, der die Verantwortung für die Durchführung der eingegangenen Verpflichtungen übernimmt.

Speziell im Hinblick auf soziale und wirtschaftliche Rechte kommt hinzu, dass der Erfüllungsdruck der Gegenseitigkeit nicht zum Zuge kommen kann. Diese Rechte begründen Verpflichtungen des Staates gegenüber seinen eigenen Bürgern. Hier braucht es nach dem Grundsatz der Subsidiarität primär innerstaatlich wirksame Durchsetzungsmechanismen, die traditionsgemäß für die klassischen Freiheitsrechte vorhanden sind, aber im Hinblick auf die Rechte der zweiten Generation nur bruchstückhaft existieren. Auch internationale Beschwerdeverfahren bringen durchweg kaum Abhilfe, weil der Schwerpunkt nicht

42 Vgl. insbesondere den 2005 World Summit Outcome, GV-Resolution 60/1, 16.9.2005, para. 121.

43 Dazu Christian Tomuschat, Human Rights - Between Idealism and Realism, 3. Aufl. (Oxford: Oxford University Press, 2014) 4. 
auf dem Unrecht im Einzelfall liegt, sondern von einer defizitären Gesamtlage herrührt. Dies haben sowohl das allgemeine Publikum wie auch die verantwortlichen Regierungen durchweg verstanden. So hat das Beschwerdeverfahren nach dem Internationalen Pakt über wirtschaftliche, soziale und kulturelle Rechte, dessen Einführung durch das Fakultativprotokoll zum Pakt im Jahre 2008 lebhaft begrüßt worden war, bisher nur 22 Ratifikationen erhalten. In der Tat ist nicht ersichtlich, inwiefern etwa eine hohe Arbeitslosigkeit in einem Lande eine spezifische Rechtsverletzung einem Arbeitsuchenden gegenüber bedeuten könnte. Hier sind zupackende Berichtsprüfungsverfahren, welche die tieferen Gründe für die Notlage zu erforschen suchen, das bessere Gegenmittel. Aber die Schlussfolgerungen des Kontrollgremiums, des Ausschusses für wirtschaftliche, soziale und kulturelle Rechte, bleiben im Status der bloßen Empfehlung stecken und werden von den Regierungen meist nur mit mildem Interesse zur Kenntnis genommen. Auch im Verfahren des Universal Periodic Review, dem sich alle Mitgliedstaaten der Vereinten Nationen stellen, ${ }^{*} 4$ spielen die Rechte der zweiten Generation meist nur eine zweitrangige Rolle, weil sich alle zur Überprüfung berufenen Mitgliedstaaten der Weltorganisation darüber einig sind, dass es ja grundsätzlich im Interesse jeder Regierung liegt, die Angehörigen des eigenen Volkes angemessen mit sozialen Dienstleistungen zu versorgen - ausgenommen die Fälle, wo eine korrupte Führungselite das eigene Volks nur als Objekt der Ausbeutung sieht. Man geht also davon aus, dass allein schon der Druck von unten die Regierung zwingt, ihren wirtschaftlichen und sozialen Verpflichtungen nachzukommen.

Nicht gelöst ist schließlich das Migrationsproblem. Hier stehen auf der einen Seite staatliche Souveränität, die das Recht zur Entscheidung über Einreise und Aufenthalt fremder Staatsangehöriger für sich in Anspruch nimmt, und das wenig konturierte Prinzip der internationalen Solidarität einander gegenüber. Ein individuelles Recht auf Asyl kennt das Völkerrecht nicht, nur die vage Aussage in Art. 14 der Allgemeinen Erklärung der Menschenrechte, dass jeder Mensch das Recht habe, „in anderen Ländern vor Verfolgung Asyl zu suchen und zu genießen“. Staatszerfall und Überbevölkerung als Fluchtursachen lassen sich vom Völkerrecht her nur in bescheidenem Umfang bekämpfen. Die internationale Gemeinschaft hat bisher weder die Kraft noch die Handlungsmittel, um einem Versagen bei der Ausübung des nationalen Selbstbestimmungsrechts, das wesentlich Selbstverantwortung für das eigene Schicksal heißt, entgegenzusteuern. ${ }^{*} 45$

\section{Schlussbemerkungen}

Zum Schluss sei der Blick nochmals auf das Grundproblem von Krieg und Frieden gelenkt. Das Völkerrecht hat hier seine optimale Form gefunden mit dem allgemeinen Gewaltverbot und der Einschränkung der legalen Gewaltanwendung auf den Fall der Selbstverteidigung und die Ermächtigung durch den Sicherheitsrat. Nicht ausgeführt zu werden braucht, dass diese grobmaschigen Regeln im Detail zu vielfältigen Auseinandersetzungen im Detail geführt haben. Aber im Grundsatz haben sie sich bewährt, auch wenn ihre Anwendung durch den Sicherheitsrat schwere strukturelle Mängel aufweist. Diese institutionelle Seite verlangt nach Verbesserung, ohne dass man sich der Täuschung hingeben sollte, dass vom normativen Standpunkt aus ein Patentrezept gefunden werden könnte. Das Völkerrecht lässt sich nicht in die Wissenschaft von den internationalen Beziehungen umwandeln. An eine Abschaffung des Vetorechts ist in der Gegenwart nicht ernsthaft zu denken. Keine der ständigen Ratsmächte ist bereit, an ihre privilegierte Stellung rühren zu lassen. Wünschenswert ist indes eine bessere Abstimmung zwischen Sicherheitsrat und Generalversammlung, damit der Gemeinwille der internationalen Gemeinschaft besser zur Geltung kommt. Weitergehende Ratschläge können vom Boden des Rechts aus nicht gemacht werden. Es liegt auf der Hand, dass internationale Spannungsursachen möglichst frühzeitig in geeigneten Verfahren ausgeräumt werden sollten. Aber menschliche Machtgier und Irrationalität lassen sich durch das Recht nicht in Fesseln legen.

Allerdings darf man auch darauf setzen, dass die in der Charta selbst und in den Menschenrechtspakten verankerten Gemeinwohlvorstellungen durch dauernde Bekräftigung von Seiten der verantwortlichen Amtsträger eine die Wirklichkeit prägende Kraft entfalten werden. Diese Hoffnung erscheint schon

4 GV-Resolution 60/251, 15.3.2006.

45 Vgl. die Erklärung über territoriales Asyl, GV-Resolution 2312(XXII), 14.12.1967 sowie die vor kurzem verabschiedete New Yorker Erklärung über Flüchtlinge und Migranten, GV-Resolution 71/1, 19.9.2016. 
allein deswegen nicht abwegig, weil ja ganz offensichtlich die Menschheit vor Entwicklungen steht, die ihre Existenz bedrohen können. Sie wird sich zunehmend als eine Notgemeinschaft begreifen müssen, die ihre Zukunft nur durch solidarisches Zusammenstehen sichern kann. Vor allem die prosperierenden Staaten des Westens - und auch diejenigen des Nahen Ostens - werden noch weit stärkere Opfer als bisher erbringen müssen, um die Armutskluft zwischen einem industriell entwickelten Norden und einem jedenfalls technologisch rückständigen Süden zu schließen. Der Gefahr eines aus der Verzweiflung der Not erwachsenden Krieges sollte man ernsthaft ins Auge blicken, um solche zerstörerischen Entwicklungen abzuwenden. Frieden bleibt eine konkrete Utopie, nicht ein inhaltsleerer Wunschtraum. ${ }^{*} 46$ Aber damit sind die Grenzen des Völkerrechts eindeutig überschritten. Es gehört zu den Aufgaben der Politik und kann nicht von der Rechtswissenschaft geleistet werden, solche Herausforderungen zu bewältigen und die Zukunft aktiv zu gestalten.

46 Zum Gedanken der konkreten Utopie vgl. Karl Mannheim, Ideologie und Utopie, 9. Aufl. (Frankfurt: Vittorio Klostermann, 2015) 169-184. Auch Ernst Bloch hat in seinem Werk immer wieder die Notwendigkeit einer „konkreten“ Utopie betont, vgl. etwa: 'Widerstand und Friede', in: Politische Messungen, Pestzeit, Vormärz [Werke, Gesamtausgabe, Bd. 11] (Frankfurt/ Main: Suhrkamp, 1970) 375, 457. 